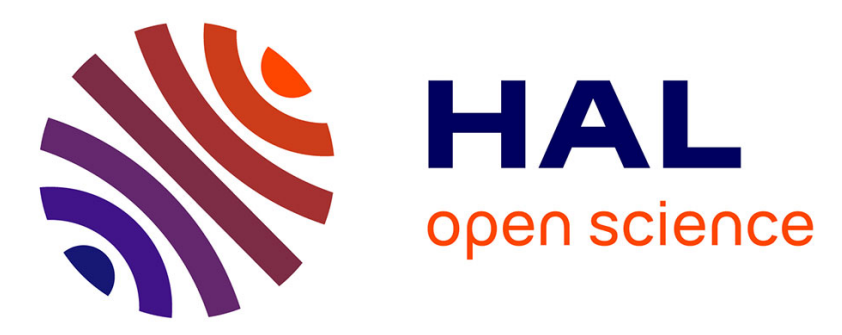

\title{
Integrated digital image correlation applied to elasto-plastic identification in a biaxial experiment
} Morgan Bertin, Francois Hild, Stéphane Roux, Florent Mathieu, Hugo Leclerc, Patrick Aimedieu

\section{- To cite this version:}

Morgan Bertin, Francois Hild, Stéphane Roux, Florent Mathieu, Hugo Leclerc, et al.. Integrated digital image correlation applied to elasto-plastic identification in a biaxial experiment. Journal of Strain Analysis for Engineering Design, 2016, 51 (2), pp.118-131. 10.1177/0309324715614759 . hal01303519

\section{HAL Id: hal-01303519 \\ https://hal.science/hal-01303519}

Submitted on 19 Apr 2016

HAL is a multi-disciplinary open access archive for the deposit and dissemination of scientific research documents, whether they are published or not. The documents may come from teaching and research institutions in France or abroad, or from public or private research centers.
L'archive ouverte pluridisciplinaire HAL, est destinée au dépôt et à la diffusion de documents scientifiques de niveau recherche, publiés ou non, émanant des établissements d'enseignement et de recherche français ou étrangers, des laboratoires publics ou privés. 


\title{
Integrated Digital Image Correlation applied to elasto-plastic identification in a biaxial experiment
}

\author{
Morgan Bertin, François Hild, ${ }^{*}$ Stéphane Roux, Florent Mathieu, Hugo \\ Leclerc and Patrick Aimedieu \\ LMT (ENS Cachan, CNRS, Université Paris-Saclay) \\ 61 avenue du Président Wilson, 94235 Cachan, France
}

\begin{abstract}
The identification of the parameters of several constitutive laws is performed with the Integrated Digital Image Correlation (IDIC) technique in a biaxial experiment for a cruciform specimen made of stainless steel. The sought material parameters are assessed with the contribution of both reaction forces (from load sensors) and displacement fields (measured via digital image correlation). For each constitutive law a global residual quantifying the model error is assessed. Keywords: Digital Image Correlation (DIC), Full field measurements, Identification, Constitutive law
\end{abstract}

\section{Introduction}

The identification and validation of constitutive models are crucial issues for mechanical design. Challenges such as optimizing structures with respect to

\footnotetext{
${ }^{*}$ Corresponding author

Email address: \{morgan.bertin; francois.hild;

stephane.roux;florent.mathieu; hugo.leclerc\}@lmt.ens-cachan.fr and patrick.aimedieu@enpc.fr. (Morgan Bertin, François Hild, ${ }^{*}$ Stéphane Roux, Florent Mathieu, Hugo Leclerc and Patrick Aimedieu)
} 
mass or using innovative materials, e.g., high performance steels or composite materials push toward complex and multiaxial constitutive models. However, standard tests [1] related to the identification of material parameters require sample geometries for which the mechanical response must be homogeneous and uniaxial. Such limitations lead to numerous elementary tests even though more complex (i.e., multiaxial) tests individually provide much more data. To characterize the latter ones, inverse identification methods based on full field measurements [2, 3] are developed. Since Hill 4] proposed to account for anisotropic plasticity, several identification strategies have been proposed. After briefly reviewing some of them, the paper seeks to solve the problematic of the identification of elastoplastic laws with Integrated Digital Image Correlation (IDIC).

First the simplest identification is performed by tuning the constitutive parameters with uniaxial strain-stress curves and least-squares fit [1]. However, this strategy does not provide internal checks of the accuracy of the resultant material parameters. Furthermore, it is assumed that the sample geometry behaves under statically determinate stress states [5]. This hypothesis is in opposition with the observed mechanical behaviors when complex and multiaxial experiments are performed.

Second approximately fifteen years after the appearance of the Finite Element Method, Kavanagh and Clough [6] proposed the Finite Element Model Updating (FEMU) technique. They focused their work on the characterization of nonlinear elastic materials. FEMU consists of minimizing the sum of squared 
residuals, a residual being the difference between the numerical and experimental displacement fields (i.e., FEMU-U), strain data (i.e., FEMU- $\epsilon$ ), load levels (i.e., FEMU-F), or combinations of the previous quantities (e.g., FEMU-UF) 3]. This technique also provided new routes to tackle two main issues, namely, i) the internal validation of the identified parameters, and $i i$ ) the use of statically indeterminate stress conditions.

Some authors proposed strategies aiming to assess elastoplastic properties of aluminium alloys [7] and metals [8] while combining experimental load measurements and FE simulations. However, full-field measurement techniques were not used and only reaction forces were considered to solve the identification problem.

The advent of DIC as an experimental tool [9] has allowed elastic properties to be determined 10, 11, 12. New strategies have been designed to tackle the increasing number of experimental data. Avril et al. 2] summarize several techniques based on full-field measurements to identify isotropic linear elastic properties. Some of these identification strategies have been extended to elastoplasticity and nonlinear mechanical behaviors. Lecompte et al. [13] identified Hill's parameters 4 ] with the FEMU technique under biaxial experiments. Conversely, Grédiac and Pierron 14 have used the virtual fields method. The constitutive error gap was also extended to plasticity [15]. Haddadi and Belhabib [16] have investigated the characterization of a hardening law on a heterogenous tensile test using the weighted-FEMU technique with both reaction force and strain field. Réthoré et al. 17] considered quasi-3D measurements with stereo digital image correlation to perform the identification of elasto-plastic constitutive pa- 
rameters using the integrated digital image correlation technique. Schmaltz et al. [18] made use of stereo-DIC measurements and FEMU-U to identify a plastic law for four different biaxial cross-shaped geometries.

All the previous methods use as input the measured displacement fields or derived strain fields. However, these kinematic fields may be seen as parameterized by the sought material properties. Such observation enables the mechanical identification to be integrated within the digital image correlation procedure. This method is called Integrated-DIC [11, 19, 20]. The latter is a global DIC technique that relies on a mechanically driven choice for the kinematic basis. The measurement and identification of the sought parameters is thus performed in a single step. Since the identification is formulated at the pixel level, the process becomes mesh independent, provided the latter allows for a faithful description of the displacement fields as obtained from the mechanical modelling [21].

The same type of integrated strategy will be followed herein. In the approach followed herein, a commercial FE package will be used in a non-intrusive way to estimate the displacement and load sensitivities to the sought material parameters. Consequently, any built-in or user-defined material model can be probed. This type of implementation makes the present work very generic. Consequently different constitutive postulates will be assessed hereafter (e.g., linear and nonlinear kinematic hardening) to discuss model errors. The analysis will deal with a cruciform specimen in a biaxial experiment under load-controlled mode. The testing machine is a compact $\left(2000 \mathrm{~cm}^{3}\right)$ biaxial device. The selected 
material is a precipitation-hardened stainless steel (i.e., 17-7 PH grade 22]). It is used thanks to its excellent mechanical properties with respect to corrosion and fatigue [23, 24]. One key aspect of the present study is related to the small (i.e., sub-millimetric) thickness of the tested sheet since it will be used to make bellows.

The paper is divided into three main sections. In Section 2 , the experimental tools and the theoretical background are presented. Integrated Digital Image Correlation (IDIC) and the chosen constitutive laws are also introduced. Section 3 deals with the experimental procedures. The sample geometry, the prescribed loads and the sources of uncertainties are assessed. Section 4 shows the identification results and a discussion based upon the identification residuals is proposed. 


\section{Experimental protocol}

The experimental procedure utilizes an electromechanical testing machine, Mini-ASTREE (Figure 1), and one digital camera with a telecentric lens. The machine prescribes arbitrary displacement and forces in two orthogonal directions, $\boldsymbol{e}_{\mathbf{1}}$ and $\boldsymbol{e}_{\mathbf{2}}$, with opposite actuators that can be controlled in a symmetric fashion so that the specimen center is motionless [25]. $\quad F_{1}$ will denote the load amplitude applied by the two coupled actuators along direction $\boldsymbol{e}_{\mathbf{1}}$, and $F_{2}$ for the other two coupled actuators. Biaxial compressive and tensile tests can be run with a load range $F_{1,2} \in[-2000 ; 2000]$ N. Furthermore the stroke rate varies from $30 \mu \mathrm{m} / \mathrm{min}$ to $5 \mathrm{~mm} / \mathrm{min}$. 16-bit gray scale images are captured with a pco.edge camera. The effective magnification is $13.5 \mu \mathrm{m}$ per pixel or $74 \mathrm{pixel} / \mathrm{mm}$.

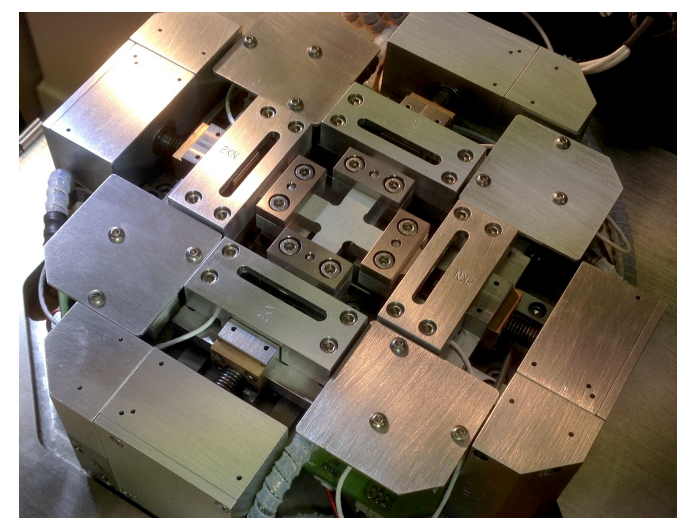

Figure 1: Biaxial testing machine. A cross-shaped sample (center of picture) has been positioned after being patterned for DIC purposes

The specimen is machined via Electrical Discharge Machining (EDM). Fig- 
ure 2(a) shows the sample geometry. The holes in the four arms allow for the positioning of the sample in the grips. This geometry is different from those analyzed by Schmaltz et al. [18. The machining process induces very small residual stresses in the specimen since no physical contact between the wire and the specimen is required. The sample is machined from a $300 \mu \mathrm{m}$ thick sheet made of precipitation hardened stainless steel (i.e., 17-7 PH grade), itself cut from a hot rolled 3-mm thick sheet. The chemical composition of the alloy is shown in Table1 1 To avoid deflection during machining the sheet is maintained between two 3-mm thick aluminum sheets. The mechanical properties given by the manufacturer are the $0.2 \%$ yield stress $\left(\sigma_{0}^{0.2 \%}=300 \mathrm{MPa}\right)$ and Poisson's ratio $(\nu=0.3)$. The Young's modulus is not mentioned and its value is assumed to be equal to $E=200 \mathrm{GPa}$, which is the value of the untreated steel.

Table 1: Chemical composition of 17-7 PH grade [22]

\begin{tabular}{|c|cccccccc|}
\hline Composition & $\mathrm{C}$ & $\mathrm{Mg}$ & $\mathrm{P}$ & $\mathrm{S}$ & $\mathrm{Si}$ & $\mathrm{Cr}$ & $\mathrm{Ni}$ & $\mathrm{Al}$ \\
\hline$($ wt $\%)$ & 0.09 & 1.00 & 0.040 & 0.030 & 1.00 & $16.00-18.00$ & $6.50-7.75$ & $0.75-1.50$ \\
\hline
\end{tabular}

The loading history may influence the identification results. A so-called triangular loading path is prescribed. Since the assessment of elastoplastic laws with kinematic hardening postulates is one of the present objectives, a cyclic loading history is prescribed. Figure 2(b) shows the $F_{2}$ versus $F_{1}$ cycles when the load amplitude is increased by $200 \mathrm{~N}$ between each of them. Furthermore, the load speed is constant and equal to $\dot{F}_{1}=\dot{F}_{2}=3000 \mathrm{~N} / \mathrm{min}$ when different from 0 . 


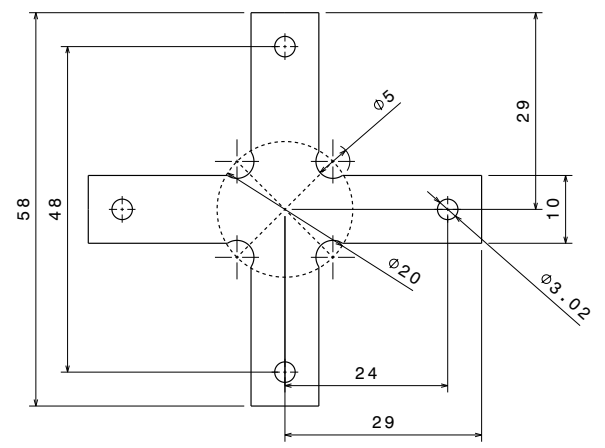

(a)

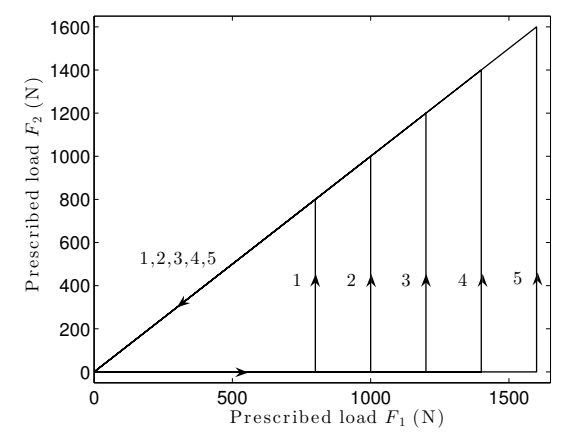

(b)

Figure 2: (a) specimen geometry (expressed in $\mathrm{mm}$ ). (b) Prescribed loading history $F_{2}$ vs. $F_{1}$ consisting of successive triangles of increasing amplitudes, and where each triangle consists of a first increase of $F_{1}$ at $F_{2}=0$, followed by an equal increase of $F_{2}$ at fixed $F_{1}$, and finally and equibiaxial unloading down to $F_{1}=F_{2}=0$

Figure 3(a) shows the Region Of Interest (ROI) that contains the specimen arms and the three-noded triangular mesh with linear interpolation (T3) used for DIC and IDIC purposes. Since FE-based DIC analyses are carried out, 
the mesh is constructed from the reference image. Consequently, it follows very faithfully the boundaries of the sample. Further, a small element size was selected (i.e., 25 pixels) to capture the strain gradients as best as possible for regular T3-DIC. For the integrated approach, it could have been further refined [19, 21, but this option was not considered herein since both approaches are to be compared with the same underlying mesh. In the following the geometric coordinates shown in Figure 3(b) will be identical for any shown field.

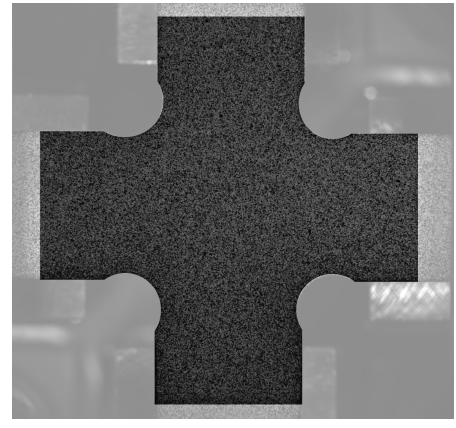

(a)

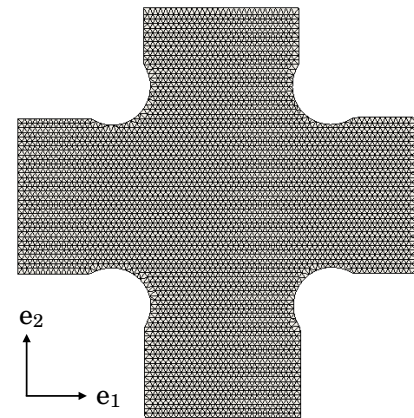

(b)

Figure 3: (a) Reference image $f$ with the Region Of Interest (ROI) in dark and (b) mesh used for DIC and IDIC analyses (the characteristic element size is 25 pixel wide) 


\section{Identification strategy}

The numerical tools and the identification strategy are summarized hereafter. First, global DIC and Integrated-DIC are formulated. Second, the constitutive laws and their respective material parameters are introduced. Last, the numerical implementation of the identification scheme is briefly recalled.

\subsection{Digital Image Correlation (DIC)}

Global DIC will be used for comparison purposes with Integrated-DIC. Among various kinematic bases, finite element shape functions have initially been introduced to deal with regular meshes made of four-noded quadrilaterals [26]. It was subsequently generalized to deal with unstructured meshes made of three-noded triangle (T3) elements [19]. DIC relies on the registration of an image $f$ in the reference configuration and a series of pictures $g$ in the deformed configurations. The problem consists of minimizing the global correlation resid-

uals $\chi_{f}^{2}=1 / N_{t} \sum_{t=1}^{N_{t}} \chi_{f t}^{2}(t)$, which is the sum of squared differences between the deformed image corrected by the measured displacement $\boldsymbol{u}(\boldsymbol{x}, t)$ and the reference image (written for each time $t$ independently) over the Region Of Interest

$$
\chi_{f t}^{2}(t)=\frac{1}{2 \gamma_{f}^{2} N_{\Omega}} \sum_{\Omega}\left((g(\boldsymbol{x}+\boldsymbol{u}(\boldsymbol{x}, t), t)-f(\boldsymbol{x}))^{2}\right.
$$

with respect to the sought displacement fields $\boldsymbol{u}(\boldsymbol{x}, t)$, where $\boldsymbol{x}$ is any considered pixel. In this expression $\Omega$ denotes the Region of Interest (ROI), $N_{\Omega}$ its area expressed in terms of the number of pixels it contains, $N_{t}$ the number of time steps, and $\gamma_{f}$ the standard deviation (expressed in gray levels) of the white 
noise assumed to affect each image independently (including the reference one, which is responsible for the factor of $1 / 2$ coming as a multiplicative term in this functional).

The displacement field is decomposed onto a basis of fields $\boldsymbol{\psi}_{n}(\boldsymbol{x})$ that is selected at will

$$
\boldsymbol{u}(\boldsymbol{x})=\sum_{n} u_{n} \boldsymbol{\psi}_{n}(\boldsymbol{x})
$$

where $\boldsymbol{\psi}_{n}$ are (vector) T3 shape functions in the present case ((i.e., three noded triangles with a linear interpolation of displacements), and $u_{n}$ the unknown degrees of freedom. Ideally, the number of these fields should be kept as small as possible (i.e., to reduce the uncertainty 1 on their amplitude) yet large enough to capture the anticipated variety or heterogeneity of the actual experimental field. The minimization of $\chi_{f}^{2}$ is achieved by successive linearizations and corrections using Newton-Raphson's scheme

$$
[\boldsymbol{M}]\{\delta \boldsymbol{u}\}=\{\boldsymbol{b}\}
$$

where $[\boldsymbol{M}]$ is the DIC matrix, $\{\delta \boldsymbol{u}\}$ the vector gathering all increments of measured displacement amplitudes, and $\{\boldsymbol{b}\}$ the residual column vector. In the present case, the minimization is performed independently for each considered time, i.e., on $\chi_{f t}^{2}$.

\footnotetext{
1 'The uncertainty $[\ldots]$ is a parameter, associated with the result of a measurement, that characterises the dispersion of the values [...] [27].' In the present study, because the sole considered origin of uncertainty is that resulting from a Gaussian white noise, the uncertainty is quantified by the standard deviation of the concerned quantity.
} 


\subsection{Integrated $D I C$}

The integration of the mechanical identification at the measurement step consists of choosing as the generalized degrees of freedom the sought parameters associated with a chosen constitutive law. Consequently, the kinematic basis is chosen as the set of sensitivity fields gathered in a matrix $\left[\boldsymbol{S}_{U}\right]$ 28]

$$
\left[\boldsymbol{S}_{\boldsymbol{U}}\right]=\frac{\partial\{\boldsymbol{u}\}}{\partial\{\boldsymbol{p}\}}
$$

where $\{\boldsymbol{u}\}$ is the computed nodal displacement vector, $\{\boldsymbol{p}\}$ the vector gathering the sought parameters in the computation via forward finite differences in the present case. One of the interests offered by an integrated approach is that the mesh size does not prevent the convergence of computations since the sought material parameters are significantly fewer in comparison with the degrees of freedom associated with the underlying FE discretization [19, 21]. Consequently, errors coming from a coarse mesh can be avoided.

If the same mesh is used, and the DIC matrix has been computed as $[\boldsymbol{M}]$, and the residual vector $\{\boldsymbol{b}\}$, the IDIC procedure simply consists of projecting the nodal displacement field onto the sensitivity fields [20]. One main difference with the previous T3-DIC procedure is that only one global minimization is performed in a single spatiotemporal analysis [29]. The identification of the sought parameters is achieved by solving iteratively until convergence linear systems

$$
\{\delta \boldsymbol{p}\}=\frac{1}{2 \gamma_{f}^{2}}\left[\boldsymbol{M}_{I D I C}\right]^{-1}\left[\boldsymbol{S}_{\boldsymbol{U}}\right]^{t}\{\boldsymbol{b}\}
$$

where $\left[\boldsymbol{M}_{I D I C}\right]=1 /\left(2 \gamma_{f}^{2}\right)\left[\boldsymbol{S}_{\boldsymbol{U}}\right]^{t}[\boldsymbol{M}]\left[\boldsymbol{S}_{\boldsymbol{U}}\right]$ is the weighted kinematic Hessian. If 
this unique quantity $\chi_{f}^{2}$ is minimized, it can be shown that it is equivalent to a weighted FEMU-U procedure provided the noise level is small [20].

The applied load is also of importance for the identification because it provides an additional measured quantity, different in nature from the kinematics, and hence it contributes significantly to the identification and reduces the uncertainty of the determined material parameters. The reaction forces can also be measured and computed. Thus, the same approach is followed with the reaction forces for which $\chi_{F}^{2}$ is minimized

$$
\chi_{F}^{2}=\frac{1}{N_{F} N_{t}}\left\{\boldsymbol{F}_{m}-\boldsymbol{F}_{c}\right\}^{t}\left[\boldsymbol{C}_{\boldsymbol{F}}\right]^{-1}\left\{\boldsymbol{F}_{m}-\boldsymbol{F}_{c}\right\}
$$

where $\left\{\boldsymbol{F}_{m}\right\}$ are the measured reaction forces and $\left\{\boldsymbol{F}_{c}\right\}$ are the computed levels with respect to the chosen material parameter set, $\left[\boldsymbol{C}_{\boldsymbol{F}}\right]$ the covariance matrix of the measured loads (in the present case it is assumed that the load measurements are uncorrelated so that $\left[\boldsymbol{C}_{\boldsymbol{F}}\right]=\gamma_{F}^{2}[\boldsymbol{I}]$, and $N_{F}$ the number of load data.

It is assumed that the load uncertainty is proportional to the magnitude of the load, namely, $\gamma_{F}^{2}=\rho_{1}^{2}|\boldsymbol{F}|^{2}$. Moreover, it is easy at this stage to incorporate a minimum measurement uncertainty for the load cells by including an additional noise term whose variance $\rho_{0}^{2}$ is independent of the load level. This practically disqualifies all measurements of forces below $F_{\min }=\rho_{0} / \rho_{1}$. Thus, in the following it is assumed that

$$
\gamma_{F}^{2}=\rho_{1}^{2}|\boldsymbol{F}|^{2}+\rho_{0}^{2}
$$

In the proposed procedure, the measured forces will be compared to the computed ones based on a numerical simulation that itself incorporates i) a 
constitutive law whose parameters will at convergence be adjusted to those of the material to be identified, and ii) Dirichlet (displacement) boundary conditions that are provided by the DIC measurements. The DIC measurement itself has an uncertainty that will induce an additional contribution, which is independent of the load magnitude as the DIC uncertainty is not dependent on the displacement amplitude, affecting computed forces rather than measured ones. However, as one will be interested in their differences, it is equivalent to transfer this additional force uncertainty on the measurement. The result is a term that cannot be distinguished from $\rho_{0}$. In practice, load cells are designed to have a dynamic range adapted to the geometry and mechanical properties of the specimen, and hence, the minimum and maximum values of these sensors are not limiting. In contrast, the identification of elastic properties requires sufficiently small strains to ensure the relevance of linear elasticity. In this case, the contribution of DIC uncertainty to the boundary conditions, and hence to the load level, is generally expected to provide a level for $\rho_{0}$ that may be limiting.

The minimization of $\chi_{F}^{2}$ leads to the variation of the identified set of material parameters

$$
\{\delta \boldsymbol{p}\}=\frac{1}{\gamma_{F}^{2}}\left[\boldsymbol{H}_{F}\right]^{-1}\left[\boldsymbol{S}_{\boldsymbol{F}}\right]^{t}\left\{\boldsymbol{F}_{m}-\boldsymbol{F}_{c}\right\}
$$

where $\left[\boldsymbol{H}_{F}\right]=\gamma_{\boldsymbol{F}}^{-2}\left[\boldsymbol{S}_{\boldsymbol{F}}\right]^{t}\left[\boldsymbol{S}_{\boldsymbol{F}}\right]$ is the static Hessian, and $\left[\boldsymbol{S}_{\boldsymbol{F}}\right]=\partial\left\{\boldsymbol{F}_{c}\right\} / \partial\{\boldsymbol{p}\}$ the reaction force sensitivities defined in the same way as the displacement field sensitivities (see Equation (3.4)). If $\chi_{F}^{2}$ is minimized alone, it corresponds to a load-based FEMU procedure, which is referred to as FEMU-F.

The identification based upon both observables, i.e., displacement field and 
reaction force, is achieved by minimizing the global functional $\chi_{I}^{2}$

$$
\chi_{I}^{2}=\frac{N_{\Omega}}{N_{\Omega}+N_{F}} \chi_{f}^{2}+\frac{N_{F}}{N_{\Omega}+N_{F}} \chi_{F}^{2}
$$

where the correlation and reaction force residuals have been introduced in Equations (3.1) and (3.6) respectively. The choice for the specific weight stems from a Bayesian foundation in the weighted quadratic difference including noise covariance. The minimization of the global residual $(3.9)$ requires an iterative computation of the parameter increments $\{\delta \boldsymbol{p}\}$

$$
\{\delta \boldsymbol{p}\}=\left[\boldsymbol{H}_{I D I C}\right]^{-1}\left(\frac{1}{2 \gamma_{f}^{2}}\left[\boldsymbol{S}_{\boldsymbol{U}}\right]^{t}\{\boldsymbol{b}\}+\frac{1}{\gamma_{F}^{2}}\left[\boldsymbol{S}_{\boldsymbol{F}}\right]^{t}\left\{\boldsymbol{F}_{m}-\boldsymbol{F}_{c}\right\}\right)
$$

where the global (i.e., kinematic and static) Hessian $\left[\boldsymbol{H}_{I D I C}\right]$ is the sum of kinematic $\left[\boldsymbol{M}_{I D I C}\right]$ and static $\left[\boldsymbol{H}_{F}\right]$ Hessians. The fact that images and load data are considered enables for the identification of the elastic parameters (i.e., the Young's modulus in particular) contrary to what was performed by Schmaltz et al. [18.

The covariance matrix of the identified parameters reads

$$
\left[\boldsymbol{C}_{p}^{I}\right]=\langle\{\delta \boldsymbol{p}\} \otimes\{\delta \boldsymbol{p}\}\rangle=\left[\boldsymbol{H}_{I D I C}\right]^{-1}
$$

where $\langle\bullet\rangle$ is the mean value of $\bullet$. Another useful indicator is the correlation matrix (no index summation used)

$$
\left(C_{0}\right)_{i j}=\frac{\left(C_{p}\right)_{i j}}{\sqrt{\left(C_{p}\right)_{i i}\left(C_{p}\right)_{j j}}}
$$

The diagonal terms of the correlation matrix are equal to 1 since one parameter is perfectly correlated with itself and off-diagonal terms vary between -1 and 1 . 


\subsection{Constitutive laws}

The objective of the present work is to assess several parameters for three constitutive laws $(A)$ linear and isotropic elasticity, and $(B, C)$ two elastoplastic laws. The latter ones correspond first to linear kinematic hardening with von Mises flow rule $(B)$, and second to an exponential kinematic hardening $(C)$ with von Mises flow rule. The total strain rate $\dot{\boldsymbol{\epsilon}}$ is written in terms of elastic and plastic strain rates $\dot{\boldsymbol{\epsilon}}=\dot{\boldsymbol{\epsilon}}_{e l}+\dot{\boldsymbol{\epsilon}}_{p l}$ where $\dot{\boldsymbol{\epsilon}}_{e l}$ is the elastic strain rate tensor and $\dot{\boldsymbol{\epsilon}}_{p l}$ the plastic strain rate tensor. The yield surface $J_{2}(\boldsymbol{\sigma}-\boldsymbol{X})=\sigma_{0}$ is defined such that $J_{2}$ is von Mises' stress, $\boldsymbol{X}$ the back-stress, and $\sigma_{0}$ the yield stress. As a first approximation, a linear kinematic hardening model is chosen for the back-stress change [30]

$$
\dot{\boldsymbol{X}}=\frac{2}{3} C \dot{\boldsymbol{\epsilon}}_{p l}
$$

where $C$ is the hardening modulus. Under the assumption of exponential kinematic hardening, the back-stress becomes

$$
\dot{\boldsymbol{X}}=\frac{2}{3} C \dot{\boldsymbol{\epsilon}}_{p l}-c \boldsymbol{X} \dot{p}
$$

where $C$ and $c$ are material parameters, $p$ the cumulative plastic strain 31 , 32.

\subsection{Numerical implementation}

The numerical procedure has been implemented in a $\mathrm{C}++$ framework, which computes the sensitivity fields from the finite element computations performed with the commercial code Abaqus [20. The IDIC code provides to the latter the required input, namely, the finite element mesh, the current values of the material parameters, the chosen constitutive law and boundary conditions obtained 
from a DIC measurement. The parameterization is based on a log scale with a ratio between the current value of the parameter and its initial guess [33]. It is defined such that the sought parameters in the new setting $\{\boldsymbol{q}\}$ are expressed from the initial basis as $\{\boldsymbol{q}\}=\log \left\{\boldsymbol{p} / \boldsymbol{p}_{\mathbf{0}}\right\}$, where $\{\boldsymbol{p}\}$ is the vector gathering the values of the parameters and $\left\{\boldsymbol{p}_{0}\right\}$ their initial values. The procedure is considered to have converged when the change in the parameters reaches a chosen threshold

$$
\|\{\delta \boldsymbol{q}\}\|_{\infty} \leq 10^{-4}
$$

where $\|\{\bullet\}\|_{\infty}$ denotes the infinite norm of the vector $\{\bullet\}$. Finally, the numerical procedure uses a Levenberg-Marquardt regularization [34, 35, which is shown to be more robust than a pure Newton based scheme [36, 7, 33] when seeking material parameters [35].

As proposed by Gras et al. [33, a regularization functional, namely $\chi_{R}$, is associated with the identification functional $\left(\chi_{I}\right)$ to enable for the evolution of the sought parameters only if their corresponding sensitivities are higher than a specific bound. This regularization prevents meaningless identification when the influences of material parameters on the observables are weak. $\chi_{R}$ is a convex function reaching its minimum equal to zero and reads

$$
\chi_{R}^{2}=\{\boldsymbol{q}\}\left[\boldsymbol{C}_{q}^{R}\right]^{-1}\{\boldsymbol{q}\}
$$

where $\left[\boldsymbol{C}_{q}^{R}\right]$ is the covariance matrix of the material parameters. Because $\chi_{I}$, and $\chi_{R}$ are both dimensionless and normalized to one their summation is valid and the regularized functional becomes $\chi^{2}=\chi_{I}^{2}+\chi_{R}^{2}$. Finally, the regularization 
of the linear system reads

$$
\left(\left[\boldsymbol{H}_{I D I C}\right]+\lambda^{*}[\boldsymbol{I}]\right)\{\delta \boldsymbol{q}\}=\left\{\boldsymbol{b}_{I D I C}\right\}+\lambda^{*}\{\boldsymbol{q}\}
$$

where $\lambda^{*}$ is the regularization parameter whose choice is performed to cancel out the influence of noise on the change of material parameters 33 .

Most of the computational cost of such integrated procedures is associated with the computation of the various sensitivities, which each time requires an additional interrogation of the FE code. For the same number of iterations IDIC and FEMU procedures lead to very similar computation times [20]. In the present case, an analysis consisting of 365 pictures required 6 hours on a PC with an 8-core Intel Xeon E5 processor for the elastoplastic nonlinear kinematic hardening law (i.e., five unknown parameters). 


\section{Identification results}

This section presents the identification results and indicators of model errors. First a resolution 2 analysis is carried out with an initial set of the sought parameters and the initial DIC solution. Second the assessment of the material parameters is performed. Last, a discussion is conducted on the kinematic, static and global residuals.

\subsection{Resolution analysis}

This part aims to estimate a priori the procedure ability to perform an identification. The DIC measurement is used to prescribe in the FEM analysis the experimentally measured displacement (Dirichlet) boundary conditions and get the sensitivity fields. Table 2 gathers the initial values of the sought material parameters. For the computation of Hessians, the parameters are ranked in the same order except for the eigenvalues ranked from smallest to largest.

Table 2: Initial value of the material parameters

\begin{tabular}{|l|c|c|c|c|c|}
\hline Parameter & $E$ & $\nu$ & $\sigma_{0}$ & $C$ & $c$ \\
\hline Value & $200 \mathrm{GPa}$ & 0.3 & $300 \mathrm{MPa}$ & $10 \mathrm{GPa}$ & 10 \\
\hline
\end{tabular}

Load uncertainties are assessed for each iterations as discussed in Section 3.1 and the displacement uncertainty is evaluated since 10 images are acquired in

\footnotetext{
2 'Resolution: smallest change in a quantity being measured that causes a perceptible change in the corresponding indication. [...] The resolution can depend on, for example, noise (internal or external) or friction.' 27]
} 
the reference configuration before loading the sample. Once the pictures have been registered, the variance $\gamma_{f}^{2}$ is estimated as the root mean square of gray level differences at convergence (Table 3).

Table 3: Standard gray level and load uncertainties

\begin{tabular}{|l|c|c|c|}
\hline Quantity & $\rho_{0}$ & $\rho_{1}$ & $\gamma_{f}$ \\
\hline Value & $2.8 \mathrm{~N}$ & $4 \times 10^{-4}$ & 302 gray levels \\
\hline
\end{tabular}

The displacement uncertainty on the constrained boundaries is evaluated by measuring the displacement fields with DIC. The covariance matrix of the unknown degrees of freedom, namely $\left[\boldsymbol{C}_{\boldsymbol{u}}\right]$ taken at the four boundaries provides this information. Furthermore, only the component in the displacement direction is kept. Therefore, the standard displacement uncertainty reads

$$
\sigma_{B C}^{2}=\frac{1}{N_{B C} \Delta t} \sum_{t}\{\boldsymbol{V}\}^{t}\left[\boldsymbol{C}_{\boldsymbol{U}}\right]\{\boldsymbol{V}\}
$$

where $\{\boldsymbol{V}\}$ is the vector that cancels out the values of the covariance matrix when they are not related to a constrained node in the prescribed displacement direction. $N_{B C}$ is the number of degrees of freedom corresponding to the boundaries. The standard uncertainty is evaluated as $\sigma_{B C}=1.3 \times 10^{-4}$ pixel. In these experimental conditions with a Young's modulus equal to $E=200 \mathrm{GPa}$, the related uncertainty provides a standard load uncertainty equal to $\rho_{0}^{D I C}=2.4 \mathrm{~N}$. Finally, the second parameter is evaluated while measuring the load with displacement control at several load levels $F \in[0 ; 2000] \mathrm{N}$. The displacement is kept constant during $300 \mathrm{~s}$. Figure 4 shows the experimental and interpolated 
standard load uncertainty. The latter depends on the applied load level and its maximum is reached for a load of $2 \mathrm{kN}$ with $\gamma_{F}=3.1 \mathrm{~N}$.

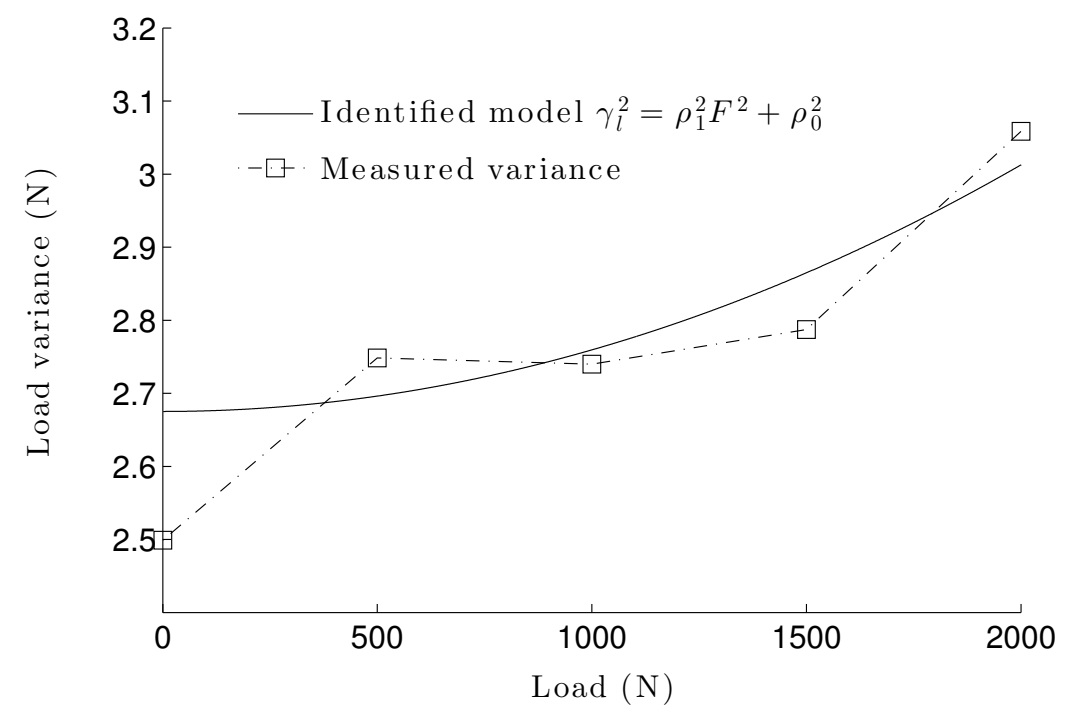

Figure 4: Measurement of the load variance under static conditions as a function of the load level. A quadratic interpolation is used [37. The value of $\rho_{0}$ is obtained when adding the uncertainty from the load cells evaluated as $\rho_{0}^{L C}=$ $0.1 \mathrm{~N}$ and that obtained from DIC $\rho_{0}^{D I C}=2.4 \mathrm{~N}$.

The evaluation of the covariance matrix (see Equation (3.11) is the key quantity to study. The Hessians are evaluated with the linear elastic law over the first load cycle, which is assumed to be essentially in that regime

$$
\left[\boldsymbol{H}_{F}\right]=\left[\begin{array}{cc}
10 & 1.4 \\
1.4 & 0.4
\end{array}\right] \times 10^{3} \quad\left[\boldsymbol{M}_{I D I C}\right]=\left[\begin{array}{cc}
0 & 0 \\
0 & 8.2
\end{array}\right] \times 10^{5} \quad\left[\boldsymbol{H}_{I D I C}\right]=\left[\begin{array}{cc}
0.1 & 0.01 \\
0.01 & 8.2
\end{array}\right] \times 10^{5}
$$

The diagonal terms of the static Hessian $\left[\boldsymbol{H}_{F}\right]$ are non-zero but separated by more than one order of magnitude. Since the Young's modulus does not influ- 
ence the kinematic fields the corresponding diagonal term $\left(M_{11}\right)$ in $\left[\boldsymbol{M}_{I D I C}\right]$ is equal to zero. The combination of both observables (see Equation (3.10) leads to $\left[\boldsymbol{H}_{I D I C}\right]$, which has nonzero diagonal terms. This last result shows that the Young's modulus is significantly easier to determine than the Poisson's ratio even when static and kinematic data are coupled.

The correlation matrix and eigenvalues are evaluated for the coupled data by resorting to $\left[\boldsymbol{H}_{I D I C}\right]$

$$
\operatorname{Cor}\left(\left[\boldsymbol{H}_{I D I C}\right]\right)=\left[\begin{array}{cc}
1.00 & 0.02 \\
0.02 & 1.00
\end{array}\right] \quad ; \quad \log _{10}\left(\lambda_{I}\right)=\left\{\begin{array}{c}
4.0 \\
5.9
\end{array}\right\}
$$

The off-diagonal terms denote a weak correlation between the two material parameters.

This first evaluation does not prove that the elastoplastic parameters can be assessed. The same approach is extended to the elastoplastic law with exponential kinematic hardening. The initial set of parameters is shown in Table 2 $\left[\boldsymbol{H}_{I D I C}\right]$ is evaluated for the first loading cycle

$$
\left[\boldsymbol{H}_{I D I C}\right]=\left[\begin{array}{ccccc}
4.5 & 0.56 & -4.2 & -0.07 & 4 \times 10^{-4} \\
0.56 & 0.87 & -0.52 & -0.01 & 6 \times 10^{-5} \\
-4.2 & -0.52 & 3.9 & 0.06 & -4 \times 10^{-4} \\
-0.07 & -0.01 & 0.06 & 0.003 & -3 \times 10^{-5} \\
4 \times 10^{-4} & 6 \times 10^{-5} & -4 \times 10^{-4} & -3 \times 10^{-5} & 3 \times 10^{-7}
\end{array}\right] \times 10^{5}
$$

and the corresponding eigen values read

$$
\log _{10}\left(\lambda_{I}\right)=\left\{\begin{array}{c}
-1.75 \\
3.23 \\
4.59 \\
5.90 \\
6.93
\end{array}\right\}
$$


Since a plastic strain occurs a variation of the Young's modulus modifies the level of the plastic behavior (i.e., for a smaller $E$ the elastic domain is greater since more strain is needed to reach the yield stress). Therefore both $\left[\boldsymbol{H}_{F}\right]$ and $\left[\boldsymbol{M}_{I D I C}\right]$ contribute for the identification of Young's modulus since both observables are influenced by the latter. Last the levels of the terms relative to $C$ and $c$ are very low denoting weak influences on the observables. The evolution of the corresponding material parameters will be avoided thanks to the regularized formulation. The corresponding correlation matrix reads

$$
\operatorname{Cor}\left(\left[\boldsymbol{H}_{I D I C}\right]\right)=\left[\begin{array}{ccccc}
1.00 & 0.28 & -0.99 & -0.58 & 0.34 \\
0.28 & 1.00 & -0.28 & -0.18 & 0.12 \\
-0.99 & -0.28 & 1.00 & 0.61 & -0.36 \\
-0.58 & -0.18 & 0.61 & 1.00 & -0.93 \\
0.34 & 0.12 & -0.36 & -0.93 & 1.00
\end{array}\right]
$$

In the early stages of plasticity the differentiation between the elastic and plastic regimes is not an easy task. Therefore when the boundary between elastic and plastic regimes are unclear the value of the yield stress depends on the Young's modulus. As a consequence, the two parameters are anticorrelated (i.e., $\left.\operatorname{Cor}\left(\left[\boldsymbol{H}_{I D I C}\right]\right)\left(E, \sigma_{0}\right) \approx-0.99\right)$. The resolution analysis over the first cycle shows that some parameters can be identified, especially elastic parameters. However, a small incursion in the plastic regime is not sufficient to identify $c$ and $C$ independently.

The same analysis is now applied for the evaluation of $\left[\boldsymbol{H}_{I D I C}\right]$ over the 
entire loading history (Figure $2(\mathrm{a})$ )

$$
\left[\boldsymbol{H}_{I D I C}\right]=\left[\begin{array}{ccccc}
89 & 4.8 & -118 & 28 & -20 \\
4.8 & 4.0 & 4.4 & -9.3 & 2.6 \\
-118 & 4.4 & 275 & -154 & 72 \\
28 & -9.3 & -154 & 125 & -51 \\
-20 & 2.7 & 72 & -51 & 28
\end{array}\right] \times 10^{5} ; \log _{10}\left(\lambda_{I}\right)=\left\{\begin{array}{c}
4.63 \\
6.42 \\
6.76 \\
7.90 \\
8.64
\end{array}\right\}
$$

The levels of the diagonal terms show that the influences of all material parameters have increased (elasto-plastic parameters included). The five eigenvalues have all their levels greater than the uncertainty and therefore can lead to the identification of all the parameters. Last the correlation matrix is evaluated from $\left[\boldsymbol{H}_{I D I C}\right]$

$$
\operatorname{Cor}\left(\left[\boldsymbol{H}_{I D I C}\right]\right)=\left[\begin{array}{ccccc}
1.00 & 0.26 & -0.75 & 0.26 & -0.40 \\
0.26 & 1.00 & 0.13 & -0.41 & 0.25 \\
-0.75 & 0.13 & 1.00 & -0.83 & 0.82 \\
0.26 & -0.41 & -0.83 & 1.00 & -0.87 \\
-0.40 & 0.25 & 0.82 & -0.87 & 1.00
\end{array}\right]
$$

still showing an important anti-correlation between the parameters $C$ and $c$. The correlation between $E$ and $\sigma_{0}$ has also been cut down.

Based on this resolution analysis two issues have been highlighted:

- Before performing the identification, it is known whether the test is discriminating to the sought parameters. In particular, it is shown that the five loading cycles are useful for the identification of the kinematic hardening parameters.

- The assessment of each parameter, its value and its uncertainty are related to the studied loading history. 
In the following the results of the identifications over the first cycle and the entire loading history are analyzed for the three constitutive laws.

\subsection{Parameter identification for the first loading step}

The material parameters are assessed for the first cycle starting from the reference set of Table 2 and with the IDIC formulation (see Equation (3.9)). For comparison purposes, a global DIC analysis is also run. In the latter no hypothesis is made on the underlying material behavior. Table 4 gathers the corresponding values and the residuals for each law at convergence. The standard uncertainties $\gamma_{p}$ are obtained from the covariance matrices $\left[\boldsymbol{H}_{I D I C}\right]$.

Table 4: Identified parameters and identification residuals for the three laws for the first cycle $\left(\gamma_{p}\right.$ is expressed in $\%$ of $\left.p\right)$.

\begin{tabular}{|c|cccccccccccccc|}
\hline law & $\chi$ & $\chi_{f}$ & $\chi_{F}$ & $E$ & $\gamma_{E}$ & $\nu$ & $\gamma_{\nu}$ & $\sigma_{0}$ & $\gamma_{\sigma_{0}}$ & $C$ & $\gamma_{C}$ & $c$ & $\gamma_{c}$ \\
& & & & $\mathrm{GPa}$ & & & & $\mathrm{MPa}$ & $\mathrm{GPa}$ & & \\
\hline$A$ & 2.10 & 2.10 & 15 & 174 & 0.4 & 0.31 & 0.09 & - & - & - & - & - & - \\
$B$ & 2.09 & 2.09 & 8.9 & 195 & 0.21 & 0.297 & 0.1 & 306 & 0.25 & 10 & - & - & - \\
$C$ & 2.09 & 2.09 & 8.9 & 195 & 0.25 & 0.297 & 0.1 & 306 & 0.3 & 10 & - & 10 & - \\
\hline
\end{tabular}

The residuals $\chi_{f}$ are close to the initial DIC residual $\left(\chi_{f}=2.06\right)$, themselves very close to the lower limit (i.e., 1) had the residuals only contained noise contributions. Figure 5 shows the change of IDIC residuals (color) and DIC residuals $\chi_{f}$ (black) with time. After the first ten pictures captured before starting the experiment the residual levels increase. The level of DIC residuals can be explained by the mesh size equal to 25 pixels, which is a compromise 
between displacement resolution and spatial resolution [38. The residual induced by the linear elastic law becomes slightly higher than the DIC residual for $t \geq 21 \mathrm{~s}$, and may point out the onset of plasticity. This is confirmed by analyzing the load residuals, which are 15 times higher than the levels expected from noise alone.

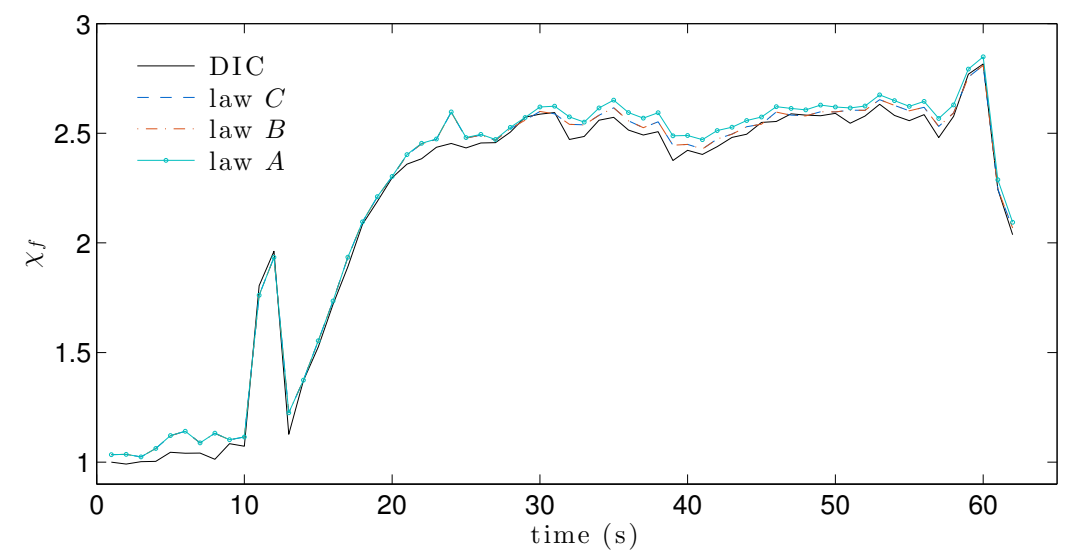

Figure 5: DIC and IDIC residuals for the three constitutive laws

Residuals induced by laws $B$ and $C$ are equivalent and marginally lower than those of law $A$. Consequently the exponential kinematic hardening does not improve the identification with respect to the experimental data. It is noteworthy that due to yielding the Young's modulus estimate is already $10 \%$ lower than the levels observed for laws $B$ and $C$. A small rise in Poisson's ratio is also observed. The level of yield stress is found to be in accordance with known values 22].

Figure 6 shows the change of the parameters with the iterations. The con- 
vergence is reached after few iterations, namely, 5,7 and 7 for laws $A, B$ and $C$ respectively. As observed in the resolution analysis, the kinematic hardening parameters keep their initial values thanks to the Levenberg-Marquardt regularization. The corresponding standard uncertainty is significantly higher for the hardening parameters than those for the elastic parameters and the yield stress (Table 4)

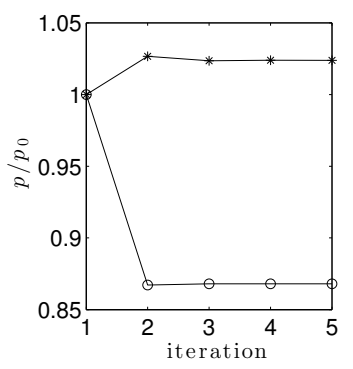

(a) Law $A$

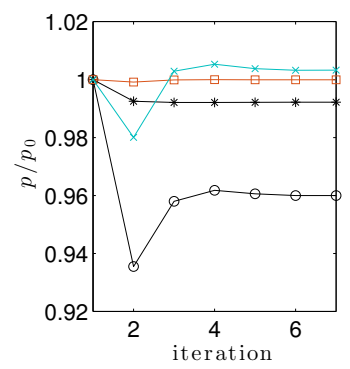

(b) Law $B$

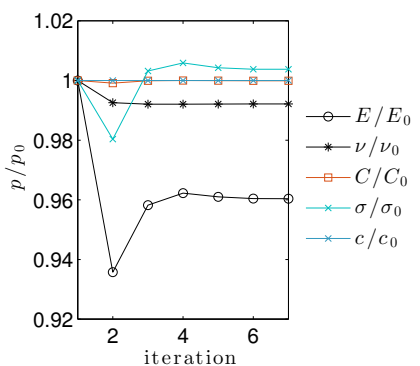

(c) Law $C$

Figure 6: Change of material parameters with the iteration number for the three investigated laws. Each parameter is normalized by its initial value

Figure 7 shows the displacement field $u_{1}$ and residual component, which is the difference between IDIC and DIC measurements at the last time step of the first load cycle $(t=61 \mathrm{~s})$ for the different constitutive postulates. Non vanishing displacements exist, which indicate the presence of plastic strains (Figure $7(a)$ ). This observation validates the results shown in Table 4 Figure 7 (b) shows that law $A$ induces very high differences in particular close to one of the connecting radii. This is an indication of plastic activity in the vicinity of stress 
concentration areas. When compared with Figures 7 (c-d) there are significant differences, again validating the identification with elastoplastic postulates that lead to equivalent differences with raw DIC. The fact that there remain displacement residuals shows that the plastic behavior has been only partially captured.

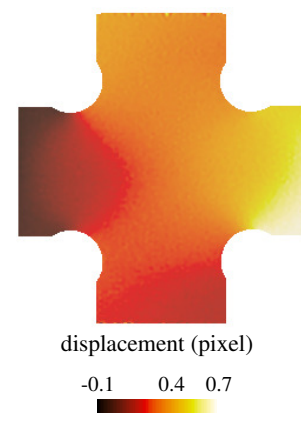

(a) DIC

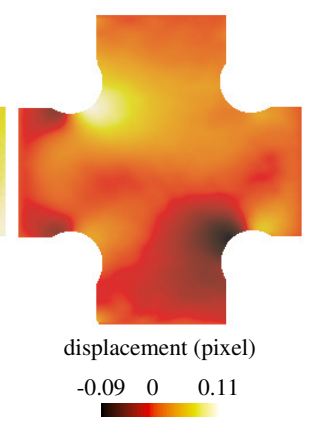

(b) DIC - IDIC $(A)$

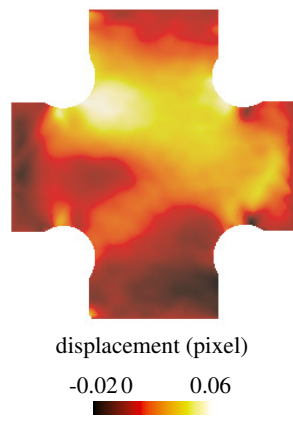

(c) DIC - IDIC $(B)$

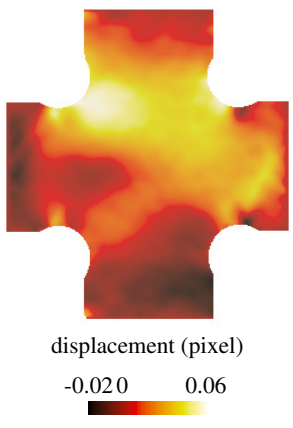

(d) DIC - IDIC $(C)$

Figure 7: Displacement field map $u_{1}$ measured by DIC and displacement differences between DIC and IDIC for the three investigated laws

\subsection{Parameter identification for the whole history}

The same analysis is now carried out over the entire loading history (Table 5). Even if the IDIC residual of law $C(\chi=6.3)$ is 2.5 times higher than the DIC residual $\left(\chi_{f}=2.5\right)$ it is the best of the three chosen postulates. The fact that the load residuals are still high is an indication that there remains a model error. This is particularly true for the elastic law that induces very high residual errors. The Young's modulus is found to be very small, and the Poisson's ratio tends to 0.5 (i.e., incompressibility as expected from dominant isochoric plasticity). 
Table 5: Identified parameters and identification residuals for the three laws for the whole loading history

\begin{tabular}{|c|cccccccccccccc|}
\hline law & $\chi$ & $\chi_{f}$ & $\chi_{F}$ & $E$ & $\gamma_{E}$ & $\nu$ & $\gamma_{\nu}$ & $\sigma_{0}$ & $\gamma_{\sigma_{0}}$ & $C$ & $\gamma_{C}$ & $c$ & $\gamma_{c}$ \\
& & & & $\mathrm{GPa}$ & & & & $\mathrm{MPa}$ & $\mathrm{GPa}$ & & \\
\hline$A$ & 15 & 14.9 & 1820 & 8.8 & 0.25 & 0.499 & 0.002 & - & - & - & - & - & - \\
$B$ & 7.1 & 7.1 & 113 & 157 & 0.15 & 0.31 & 0.04 & 480 & 0.15 & 6.7 & 0.15 & - & - \\
$C$ & 6.3 & 6.3 & 100 & 148 & 0.16 & 0.30 & 0.04 & 423 & 0.16 & 8.6 & 0.16 & 10.8 & 0.03 \\
\hline
\end{tabular}

All these trends clearly disqualify elasticity as a model able to describe the reported experiment. This is confirmed by Figure 8 that shows the change of IDIC and DIC residuals over time. Unlike the previous results (Figure 5), gaps between IDIC and DIC residuals are much more pronounced, especially when the level of plastic strain increases. Furthermore, the gain offered by each law with respect to raw DIC results is also clarified.

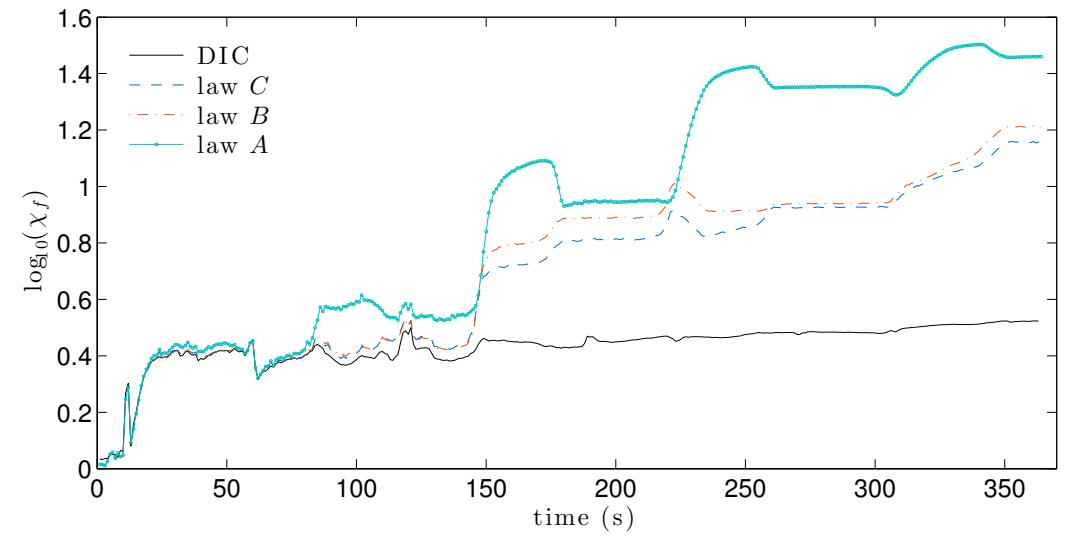

Figure 8: DIC and IDIC residual history for the three constitutive laws. Please note that a semi-logarithmic scale is used 
For law $C$, all material parameters have been assessed with a low level of uncertainty. Regarding laws $B$ and $C$ all the parameters converge in few steps (see Figure 9). Unlike Figure 6 all the parameters are modified and no regularization is needed in the present case. It is worth noting that the more freedom the identification code has (i.e., the number of unknowns is increased), the fewer iterations are needed. Further, the converged solutions are rather far from the initial guesses, which shows the robustness of the procedure.

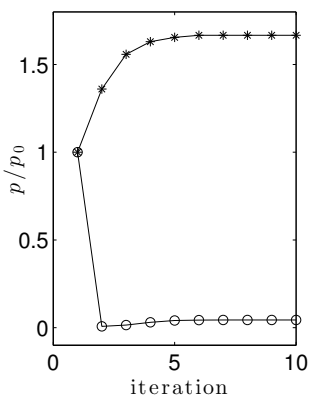

(a) Law $A$

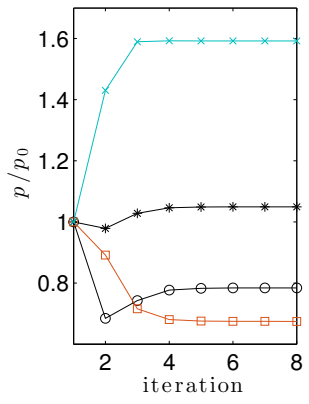

(b) Law $B$

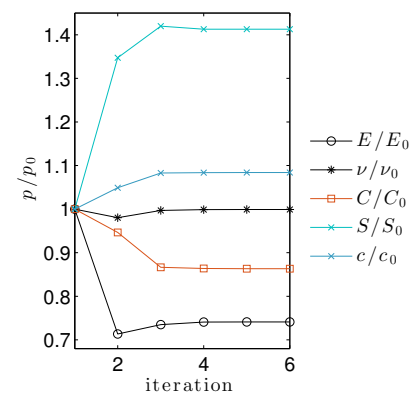

(c) Law $C$

Figure 9: Change of the material parameters for the three investigated laws during the identification iterations. Each parameter is normalized by its initial value

Figure 10 shows the displacement maps $u_{1}$ and $u_{2}$ at the last time step of the analysis. The permanent strains are important and the maximum measured eigen strain is equal to $\epsilon_{I}=18.7 \%$. The normal strain map $\epsilon_{11}$ is shown at the same time in Figure 10(c). The strain levels are the highest in the concentration zones induced by the fillets. 


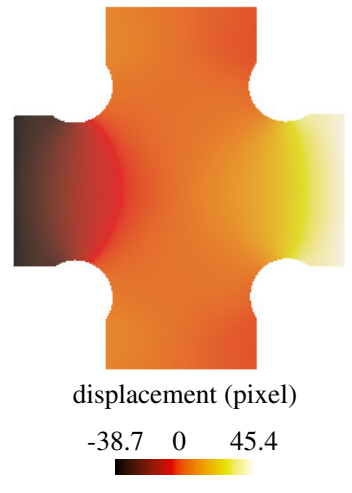

(a) Displacement $u_{1}$

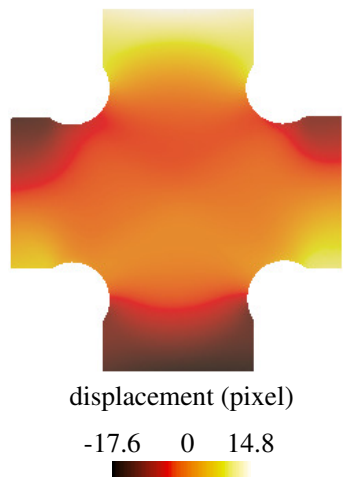

(b) Displacement $u_{2}$

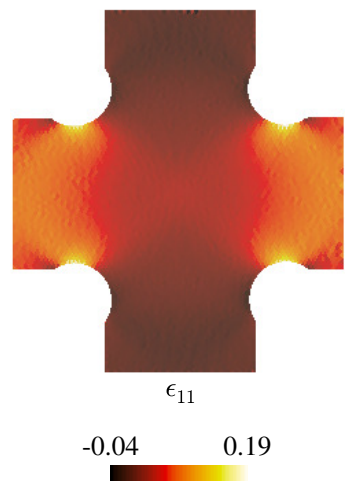

(c) Strain $\epsilon_{11}$

Figure 10: (a-b) Measured displacement fields with DIC at the last experimental time step. (c) Corresponding strain field (normal component along direction 1)

\subsection{Comparison with FEMU-F results}

Table 6 shows the parameters assessed only by considering the reaction forces (Equation (3.6). It corresponds to a FEMU-F procedure [20. To compare both approaches, the gray level and global residuals are also computed. As expected, the static residuals are lowered for all laws since the residuals only depend on reaction forces. Conversely, the overall quality degrades in addition to that associated with gray level residuals. More importantly, the standard uncertainties of the identified parameters are significantly higher, which is caused by the limited number of data used per analyzed time.

Figure 11 shows the displacement field difference for component $u_{1}$ for the three constitutive laws with IDIC or FEMU-F approaches compared with DIC measurements at the end of the experiment. The choice of an elastoplastic 
Table 6: Identified parameters and identification residuals via FEMU-F for the three laws over the entire loading history cycle (no regularization prescribed)

\begin{tabular}{|c|cccccccccccccc|}
\hline law & $\chi$ & $\chi_{f}$ & $\chi_{F}$ & $E$ & $\gamma_{E}$ & $\nu$ & $\gamma_{\nu}$ & $\sigma_{0}$ & $\gamma_{\sigma_{0}}$ & $C$ & $\gamma_{C}$ & $c$ & $\gamma_{c}$ \\
& & & & $\mathrm{GPa}$ & & & & $\mathrm{MPa}$ & $\mathrm{GPa}$ & & \\
\hline$A$ & 19.1 & 19 & 1732 & 10 & 0.4 & 0.19 & 4 & - & - & - & - & - & - \\
$B$ & 8.1 & 8.0 & 11.9 & 188 & 0.7 & 0.23 & 4 & 330 & 0.4 & 3.7 & 1.2 & - & - \\
$C$ & 7.5 & 7.5 & 9.6 & 191 & 0.7 & 0.25 & 4 & 300 & 0.5 & 8.5 & 3.7 & 29 & 11 \\
\hline
\end{tabular}

law instead of an elastic law decreases the displacement difference, thereby decreasing the model error. The residual level is lower when the identification is performed with IDIC rather than FEMU-F. An overall residual remains because $i$ ) the model does not correctly predict the plastic strains, ii) localization phenomena occur. 


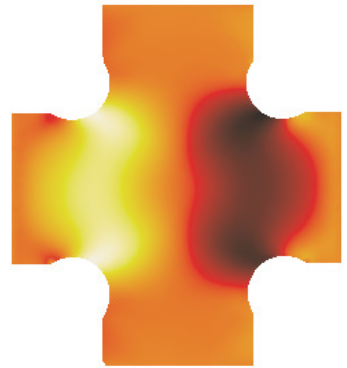

displacement (pixel)

$\begin{array}{lll}-6.47 & 0 & 5.46\end{array}$

(a) law $A$

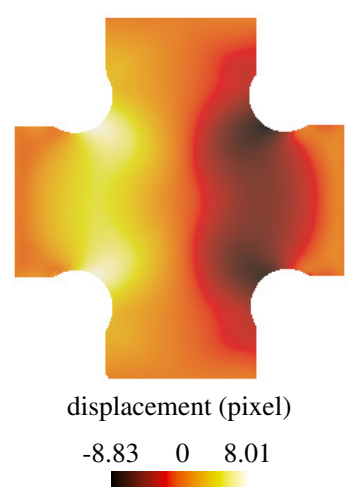

(d) law $A$

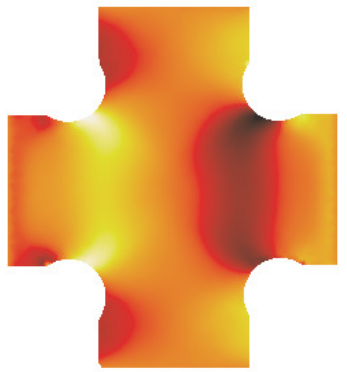

displacement (pixel)

$\begin{array}{lll}-3.00 & 0 & 2.44\end{array}$

(b) law $B$

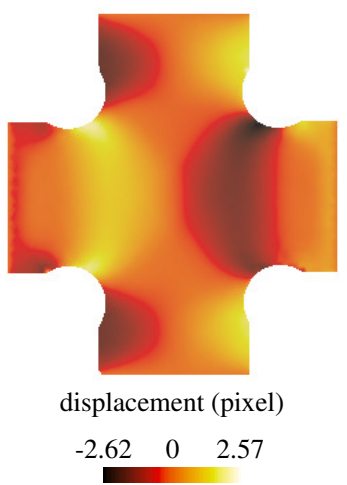

(e) law $B$

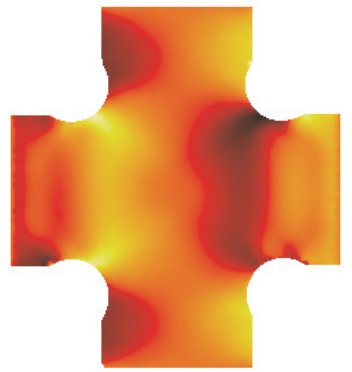

displacement (pixel) $\begin{array}{lll}-2.03 & 0 & 1.99\end{array}$

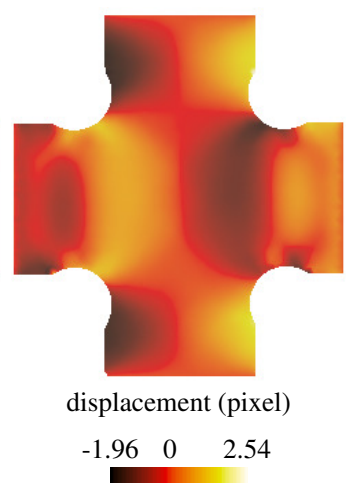

Figure 11: Displacement differences $u_{1}$ between DIC and IDIC (first row) or FEMU-F (second raw) for the three investigated laws. The color bars correspond to the largest and lowest values of the corresponding residual map 


\section{Conclusion}

Three constitutive laws have been investigated with Integrated Digital Image Correlation to analyze a biaxial test with a cruciform specimen of a thin sheet of precipitation hardened stainless steel. A general formulation was implemented to account for two sources of data (i.e., gray level picture stack and

load data). A weighting that originates from a Bayesian foundation has been followed. Consequently, each pixel and load cell play the same role, when they respective noise level is accounted for.

An initial sensitivity analysis enables the impact of the loading history and the acquisition noise on the identification to be understood. It shows that the first loading cycle is not sufficient to identify plastic laws but it is sufficient to detect its onset with the initial set of the material parameters. The corresponding identification leads to an IDIC residual with similar level as raw DIC. The identification is also performed over the entire loading history accounting for 5 loading/unloading cycles. Any of the chosen model does not match the available data with the same accuracy as observed for the first cycle. The three laws lead to three different residuals and the exponential kinematic hardening law provides the best results.

In the present study four different error indicators have been considered:

- When DIC and IDIC analyses are performed, the gap with respect to gray level conservation is computed. It estimates the registration quality when the pictures in the deformed configurations are corrected by the measured displacement field and subsequently compared with the picture 
of the reference configuration.

- DIC and IDIC can also be compared by computing the displacement differences measured by both approaches. When the mesh is identical, as in the present case, the comparison is straight forward.

- Load residuals are yet another way of characterizing the identification quality. In the present analyses, FEMU-F and IDIC could be compared since both included load residuals in their respective formulation.

- Last IDIC as developed herein has an overall quality indicator $\chi_{I D I C}^{2}$ that compares all sensor information (be it gray levels for each pixel or load level) in the same footing when normalized by the variance of corresponding noise.

This wealth of indicators allows the user to assess not only globally but also with each of the estimators the identification quality and the underlying model error (i.e., choice of constitutive law, finite element discretization).

The present study aims to pave the way to understand and design experimental protocols to not only identify but to (in)validate constitutive laws. In forthcoming works, the IDIC framework will be used to identify other laws for other materials such as polymers and composites. Other scales of observation may also be investigated by using other imaging systems such as confocal microscopy or scanning electron microscopy. 


\section{Acknowledgments}

It is a pleasure to acknowledge the support of Région Ile de France (DICCIT and THERMOFLUIDE-RT projects). 


\section{References}

[1] ISO/DIS6892-1, Metallic materials - Tensile testing - Part 1: Method of test at room temperature, International Organization for Standardization (2011).

[2] S. Avril, M. Bonnet, A. Bretelle, M. Grédiac, F. Hild, P. Ienny, F. Latourte, D. Lemosse, S. Pagano, E. Pagnacco, F. Pierron, Overview of identification methods of mechanical parameters based on full-field measurements, Experimental Mechanics 48 (2008) 381-402.

[3] M. Grédiac, F. Hild, Full-Field Measurements and Identification in Solid Mechanics, ISTE, Wiley, 2012.

URL http://books.google.fr/books?id=3bNmDdp5POkC

[4] R. Hill, A theory of the yielding and plastic flow of anisotropic metals, Proceedings of the Royal Society of London. Series A, Mathematical and Physical Sciences 193 (1033) (1948) 281-297.

[5] M. Arcan, Z. Hashin, A. Voloshin, A method to produce uniform planestress states with applications to fiber-reinforced materials, Experimental Mechanics 18 (4) (1978) 141-146. doi:10.1007/BF02324146.

URL http://dx.doi.org/10.1007/BF02324146

[6] K. T. Kavanagh, R. W. Clough, Finite element applications in the characterization of elastic solids, International Journal of Solids and Structures

7 (1) (1971) 11 - 23. doi:http://dx.doi.org/10.1016/0020-7683(71) 
$90015-1$.

URL http://www.sciencedirect.com/science/article/pii/ 0020768371900151

[7] J. Gelin, O. Ghouati, An inverse method for determining viscoplastic properties of aluminium alloys, Journal of Materials Processing Technology 45 (1-4) (1994) 435 - 440 . doi:http: //dx.doi.org/10.1016/0924-0136(94)90378-6.

URL http://wwW.sciencedirect.com/science/article/pii/ 0924013694903786

[8] O. Ghouati, J. Gelin, Identification of material parameters directly from metal forming processes, Journal of Materials Processing Technology 80-81 (0) (1998) 560 - 564. doi:http: //dx.doi.org/10.1016/S0924-0136(98)00159-9

URL http://www.sciencedirect.com/science/article/pii/ S0924013698001599

[9] M. Sutton, W. Wolters, W. Peters, W. Ranson, S. McNeill, Determination of displacements using an improved digital correlation method, Image and Vision Computing 1 (3) (1983) 133 - 139. doi:http://dx.doi.org/10.1016/0262-8856(83)90064-1.

URL http://www.sciencedirect.com/science/article/pii/ 0262885683900641

[10] G. Geymonat, F. Hild, S. Pagano, Identification of elastic parameters 
by displacement field measurement, Comptes Rendus Mécanique 330 (6)

(2002) 403 - 408. doi:http://dx.doi.org/10.1016/S1631-0721(02)

$01476-6$

URL http://www.sciencedirect.com/science/article/pii/

S1631072102014766

[11] F. Hild, S. Roux, Digital image correlation: from displacement measurement to identification of elastic properties - a review, Strain 42 (2) (2006) 69-80. doi:10.1111/j.1475-1305.2006.00258.x.

URL http://dx.doi.org/10.1111/j.1475-1305.2006.00258.x

[12] D. Lecompte, A. Smits, H. Sol, J. Vantomme, D. V. Hemelrijck, Mixed numerial-experimental technique for orthotropic parameter identification using biaxial tensile tests on cruciform specimens, International Journal of Solids and Structures 44 (2007) 1643-1656.

[13] D. Lecompte, Elastic and elasto-plastic material parameter identification by inverse modeling of static tests using digital image correlation, Ph.D. thesis, Vrije Universiteit Brussel and Koninklijke Militaire School (March 2007).

[14] M. Grédiac, F. Pierron, Applying the virtual fields method to the identification of elasto-plastic constitutive parameters, International Journal of Plasticity 22 (4) (2006) 602 - 627. doi:http: //dx.doi.org/10.1016/j.ijplas.2005.04.007. 
URL

http://www.sciencedirect.com/science/article/pii/

S0749641905000896

[15] F. Latourte, A. Chrysochoos, S. Pagano, B. Wattrisse, Elastoplastic behavior identification for heterogeneous loadings and materials, Experimental Mechanics 48 (4) (2008) 435-449. doi:10.1007/s11340-007-9088-y.

URL http://dx.doi.org/10.1007/s11340-007-9088-y

[16] H. Haddadi, S. Belhabib, Improving the characterization of a hardening law using digital image correlation over an enhanced heterogeneous tensile test, International Journal of Mechanical Sciences 62 (1) (2012) 47 - 56. doi:http://dx.doi.org/10.1016/j.ijmecsci.2012.05.012.

URL http://www.sciencedirect.com/science/article/pii/ S0020740312001221

[17] J. Réthoré, Muhibullah, T. Elguedj, M. Coret, P. Chaudet, A. Combescure, Robust identification of elasto-plastic constitutive law parameters from digital images using 3d kinematics, International Journal of Solids and Structures 50 (1) (2013) 73-85.

[18] S. Schmaltz, K. Willner, Comparison of different biaxial tests for the inverse identification of sheet steel material parameters, Strain 50 (5) (2014) 389403. doi:10.1111/str.12080.

URL http://dx.doi.org/10.1111/str.12080

[19] H. Leclerc, J. Périé, S. Roux, F. Hild, Integrated digital image correlation 
for the identification of mechanical properties, Vol. LNCS 5496, Springer, Berlin, 2009, pp. 161-171.

[20] F. Mathieu, H. Leclerc, F. Hild, S. Roux, Estimation of elastoplastic parameters via weighted femu and integrated DIC, Experimental Mechanics 55 (1) (2015) 105-119. doi:10.1007/s11340-014-9888-9.

URL http://dx.doi.org/10.1007/s11340-014-9888-9

[21] D. Lindner, F. Mathieu, F. Hild, O. Allix, C. Ha Minh, O. Paulien-Camy, On the evaluation of stress triaxiality fields in a notched titanium alloy sample via integrated digital image correlation, ASME. J. Appl. Mech. $82(7)$.

[22] AK-Steel, Mechanical characteristics 17-7 PH steel, AK-Steel (2013).

[23] A. Leybold-Herbert, Axial-load fatigue tests on 17-7 ph stainless steel under constant-amplitude loading, NASA TN D-439 62 71013, National Aeronautics and Space Administration, Langley Research Center Langley Field, Va. (October 1960).

[24] Y. Tick-Hon, Cyclic plasticity of 17-7 precipitation-hardenable semiaustenic stainless steel, Ph.D. thesis, University of Toronto (1998).

[25] J. Périé, S. Calloch, C. Cluzel, F. Hild, Analysis of a multiaxial test on a c/c composite by using digital image correlation and a damage model, Exp. Mech. 42 (3) (2002) 318-328.

[26] G. Besnard, F. Hild, S. Roux, Finite-element" displacement fields 
analysis from digital images: Application to portevin-le châtelier

bands, Experimental Mechanics 46 (6) (2006) 789-803. doi:10.1007/

s11340-006-9824-8.

URL http://dx.doi.org/10.1007/s11340-006-9824-8

[27] I. g. 99-12:2007, International Vocabulary of Metrology - Basic and General Concepts and Associated Terms, VIM, International Organization for Standardization, Geneva (Switzerland), 2007.

[28] A. Tarantola, Inverse Problems Theory. Methods for Data Fitting and Model Parameter Estimation, Elsevier Applied Science, Southampton (UK), 1987.

[29] J. Neggers, J. P. M. Hoefnagels, M. G. D. Geers, F. Hild, S. Roux, Timeresolved integrated digital image correlation, International Journal for $\mathrm{Nu}-$ merical Methods in Engineering 103 (3) (2015) 157-182.

[30] W. Prager, B. U. D. of Applied Mathematics, U. S. O. of Naval Research, U. S. N. D. B. of Ships, A New Method of Analyzing Stresses and Strains in Work-hardening Plastic Solids, Technical report (Brown University. Division of Applied Mathematics), Division of Applied Mathematics, Brown University, 1955.

URL http: //books . google.nl/books?id=UbDntgAACAAJ

[31] C. O. Frederick, P. Armstrong, A mathematical representation of the multiaxial bauschinger effect, Materials at High Temperatures 24 (1) (1966) 1-26. 
[32] J. Lemaitre, J. Chaboche, Mechanics of Solid Materials, Cambridge University Press, Cambridge (UK), 1990.

[33] R. Gras, H. Leclerc, F. Hild, S. Roux, J. Schneider, Identification of a set of macroscopic elastic parameters in a $3 \mathrm{~d}$ woven composite: Uncertainty analysis and regularization, International Journal of Solids and Structures (2014) DOI 10.1016/j.ijsolstr.2013.12.023doi: $10.1016 / j . i j$ solstr.2013.12.023

URL http://www.sciencedirect.com/science/article/pii/ S0020768313004964

[34] K. Levenberg, A method for the solution of certain non-linear problems in least squares, Quarterly Journal of Applied Mathmatics II (2) (1944) $164-168$.

[35] D. Marquardt, An algorithm for least-squares estimation of nonlinear parameters, SIAM J. Appl. Math. 11 (2) (1963) 431-441.

[36] D. S. Schnur, N. Zabaras, An inverse method for determining elastic material properties and a material interface, International Journal for Numerical Methods in Engineering 33 (10) (1992) 2039-2057. doi:10.1002/nme. 1620331004 .

URL http://dx.doi.org/10.1002/nme.1620331004

[37] cofrac, Guide technique d'accréditation: Métrologie des forces, COFRAC, lab gta 03 Edition (2003). 
[38] F. Hild, S. Roux, Comparison of local and global approaches to digital image correlation, Experimental Mechanics 52 (9) (2012) 1503-1519. doi: $10.1007 / \mathrm{s} 11340-012-9603-7$

URL http://dx.doi.org/10.1007/s11340-012-9603-7 


\section{Main notations}

\begin{tabular}{|c|c|}
\hline$\chi_{f}$ & digital image correlation objective function (DIC) \\
\hline$\chi_{\boldsymbol{F}}$ & finite element method updating objective function $(F E M U-F)$ \\
\hline$\chi_{\boldsymbol{I}}$ & Integrated Digital Image Correlation objective function (IDIC) \\
\hline$\chi_{\boldsymbol{R}}$ & Regularization objective function \\
\hline$\{\boldsymbol{p}\}$ & vector gathering constitutive parameters in the initial basis \\
\hline$\{\boldsymbol{q}\}$ & vector gathering constitutive parameters in the log basis \\
\hline $\boldsymbol{x}$ & $2 \mathrm{D}$ or $3 \mathrm{D}$ coordinates in normal space \\
\hline$e_{\alpha}$ & unit vector along direction $\alpha=1,2$ \\
\hline$\psi(\boldsymbol{x})$ & matrix gathering the shape functions of element $e$ \\
\hline$f(x), g(x)$ & pictures in the reference and deformed configurations, respectively \\
\hline$\gamma_{f}$ & standard deviation of gray levels \\
\hline$\gamma_{\boldsymbol{F}}$ & standard uncertainty of the load measurement \\
\hline$\Omega$ & region of interest \\
\hline$u(x)$ & displacement field vector \\
\hline$[M]$ & global DIC matrix \\
\hline
\end{tabular}


$\left[\boldsymbol{S}_{\boldsymbol{U}}\right] \quad$ displacement sensitivity matrix

$\left[\boldsymbol{S}_{\boldsymbol{F}}\right] \quad$ force sensitivity matrix

$N_{F} \quad$ number of load sensors

$N_{t} \quad$ number of time steps

$N_{\Omega} \quad$ number of pixels related to $|\Omega|$

$\left[\boldsymbol{C}_{p}^{\bullet}\right] \quad$ covariance matrix of identified material parameters using a method labeled by $\bullet$

$\left[\boldsymbol{M}_{I D I C}\right]$ Hessian matrix of the displacement IDIC approach

$\left[\boldsymbol{H}_{\boldsymbol{F}}\right]$ Hessian matrix of the FEMU-F approach

$\left[\boldsymbol{H}_{I D I C}\right]$ Hessian matrix of the global IDIC approach 


\section{List of Figures}

$1 \quad$ Biaxial testing machine. A cross-shaped sample (center of picture) has been positioned after being patterned for DIC purposes 6

2 (a) specimen geometry (expressed in $\mathrm{mm}$ ). (b) Prescribed loading history $F_{2}$ vs. $F_{1}$ consisting of successive triangles of increasing amplitudes, and where each triangle consists of a first increase of $F_{1}$ at $F_{2}=0$, followed by an equal increase of $F_{2}$ at fixed $F_{1}$, and finally and equibiaxial unloading down to $F_{1}=F_{2}=0 \ldots \ldots$. . 8

3 (a) Reference image $f$ with the Region Of Interest (ROI) in dark and (b) mesh used for DIC and IDIC analyses (the characteristic element size is 25 pixel wide) $\ldots \ldots \ldots \ldots \ldots$

4 Measurement of the load variance under static conditions as a function of the load level. A quadratic interpolation is used [37]. The value of $\rho_{0}$ is obtained when adding the uncertainty from the load cells evaluated as $\rho_{0}^{L C}=0.1 \mathrm{~N}$ and that obtained from DIC $\rho_{0}^{D I C}=2.4 \mathrm{~N} . \ldots \ldots \ldots \ldots \ldots \ldots \ldots \ldots \ldots \ldots \ldots \ldots$

$5 \quad$ DIC and IDIC residuals for the three constitutive laws $\ldots . .26$

$6 \quad$ Change of material parameters with the iteration number for the three investigated laws. Each parameter is normalized by its initial value $\ldots \ldots \ldots \ldots \ldots \ldots . \ldots \ldots$

$7 \quad$ Displacement field map $u_{1}$ measured by DIC and displacement differences between DIC and IDIC for the three investigated laws 28 
8 DIC and IDIC residual history for the three constitutive laws. Please note that a semi-logarithmic scale is used . . . . . . . 29

$9 \quad$ Change of the material parameters for the three investigated laws during the identification iterations. Each parameter is normalized by its initial value . . . . . . . . . . . . . . . . 30

10 (a-b) Measured displacement fields with DIC at the last experimental time step. (c) Corresponding strain field (normal component along direction 1$)$

11 Displacement differences $u_{1}$ between DIC and IDIC (first row) or FEMU-F (second raw) for the three investigated laws. The color bars correspond to the largest and lowest values of the corresponding residual map . . . . . . . . . . . . . . . . . 33 